\title{
AVALIAÇÃO DO POTENCIAL DE ADSORÇÃO DO CORANTE AZUL DE METILENO PELA CINZA LEVE PROVENIENTE DA GASEIFICAÇÃO DO CARVÃO DE CANDIOTA - RS
}

\author{
E. O. SILVA ${ }^{1}$, F. P. OLIVEIRA ${ }^{1}$, F. P. GUTERRES ${ }^{1}$, A. R. F. ALMEIDA ${ }^{1}$, \\ A. R. C. MUNIZ ${ }^{1}$ \\ ${ }^{1}$ Universidade Federal do Pampa, Curso de Engenharia Química - Bagé, RS \\ E-mail para contato: andre.almeida@ unipampa.edu.br
}

\begin{abstract}
RESUMO - O carvão rio-grandense apresenta potencial para o processo de gaseificação. Tal processo gera cinzas como resíduo. Em vista de sua grande disponibilidade, este trabalho visa o estudo do potencial de adsorção da cinza leve da gaseificação para o tratamento de efluentes. Para isto, realizaram-se ensaios de cinética e isotermas de adsorção utilizando uma solução de azul de metileno a 300 $\mathrm{mg} \mathrm{L}^{-1}$. As amostras foram agitadas, centrifugadas e, posteriormente, realizou-se a leitura das absorbâncias em espectrofotômetro UV-Vis. A partir dos dados obtidos realizaram-se ajustes aos modelos cinéticos de pseudo $1^{\mathrm{a}}$ ordem, pseudo $2^{\mathrm{a}}$ ordem e difusão intrapartícula e aos modelos de isoterma de Langmuir, Freundlich, Temkin e Sips. Para a cinética o modelo de pseudo $2^{\mathrm{a}}$ ordem apesentou um melhor ajuste com $R^{2}$ de 0,98 e $q_{e}$ de $14,3 \mathrm{mg} \mathrm{g}^{-1}$. Já para as isotermas, tanto o modelo de Langmuir quanto o de Freundlich mostraram-se satisfatórios. Ressalta-se que a cinza apresentou um potencial de adsorção com um máximo de 99,95\%.
\end{abstract}

\section{INTRODUÇÃO}

No Brasil, a maior parte das reservas de carvão mineral se encontram no sul do país. Os carvões sulbrasileiros possuem um baixo poder calorífico $(3300 \mathrm{kcal} / \mathrm{kg})$ e alto teor de enxofre (1,3\% em massa) e de cinzas (51,4\% em massa). Sendo assim, não são ideais para a utilização em termelétricas, mas são indicados para o processo de gaseificação (Garcia, 2002; Aneel, 2008; Rohde e Machado, 2016).

A gaseificação é um processo no qual o carvão reage com um oxidante resultando em um produto combustível. São gerados o syngas, alcatrão de carvão e cinzas (Smoot e Smith, 1985). As cinzas são resíduos sólidos provenientes da eliminação dos compostos voláteis do carvão e podem ser leves ou pesadas. As leves são partículas muito finas $(3-5 \mu \mathrm{m})$ que são arrastadas pelos gases durante o processo (Oka, 2004; Basu, 2006; Rohde e Machado, 2016).

O uso de resíduos industriais e orgânicos como materiais alternativos, de baixo custo, para o tratamento de efluentes tem se tornado bastante comum (Kunz e Peralta-Zamora, 2002). Tais resíduos podem ser utilizados para o tratamento de efluentes têxteis.

As indústrias têxteis geram efluentes em grandes volumes e com um alto potencial contaminante (Cardoso, 2010). A presença de corantes sintéticos nos corpos hídricos, além da 
poluição visual, causa sérios danos à fauna e à flora (Diehl et al., 2011). Além disso, muitos desses corantes podem causar problemas como alergias, dermatites e irritações cutâneas (entre elas mutagênicas e carcinogênicas) quando em contato com o ser humano (Royer, 2008). Entre esses corantes, o azul de metileno é um composto orgânico do tipo catiônico, amplamente utilizado neste tipo de indústria (Fungaro e Bruno, 2009).

Destarte, o presente trabalho objetiva o estudo do potencial de adsorção do corante azul de metileno pela cinza leve gerada no processo de gaseificação do carvão de Candiota - RS.

\section{MATERIAIS E MÉTODOS}

O Laboratório de Energia e Carboquímica, situado no campus Bagé da Universidade Federal do Pampa conduz estudos com o carvão mineral extraído das jazidas de Candiota - RS. A cinza leve proveniente da gaseificação deste carvão foi utilizada neste trabalho, a qual pode ser vista na Figura 1.

Figura 1 - Cinza leve da gaseificação do carvão de Candiota.

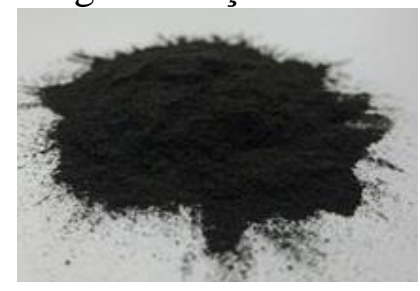

Os experimentos foram realizados utilizando o corante azul de metileno como adsorvato na concentração de $300 \mathrm{mg} \mathrm{L}^{-1}$ e todas as amostras foram utilizadas em duplicata.

Para o ensaio de cinética de adsorção utilizou-se 8 amostras com $1 \mathrm{~g}$ de cinza, colocadas em Erlenmeyer com $50 \mathrm{~mL}$ da solução de azul de metileno. As amostras foram colocadas em mesa agitadora $(150 \mathrm{rpm})$, variando-se o tempo de 10 a $175 \mathrm{~min}$. Posteriormente, foram centrifugadas por $20 \mathrm{~min}$ a $3000 \mathrm{rpm}$ e realizadas leituras de absorbância a $660 \mathrm{~nm}$ em espectrofotômetro UV-Vis. A concentração remanescente foi calculada a partir da curva de calibração para o azul de metileno, obtida previamente. Com os valores obtidos de $q_{e}$ realizouse ajustes aos modelos cinéticos de pseudo $1^{\mathrm{a}}$ ordem, pseudo $2^{\mathrm{a}}$ ordem e difusão intrapartícula. As isotermas foram obtidas por meio de 10 ensaios de adsorção variando a massa de cinza no intervalo de 0,2 a 2 g. O material adsorvente foi colocado em contato com $50 \mathrm{~mL}$ de solução de azul de metileno, agitado por $50 \mathrm{~min}$ em mesa agitadora e centrifugado por $20 \mathrm{~min}$. Realizaram-se ajustes aos modelos de isotermas de Langmuir, Freundlich, Sips e Temkin.

\section{RESULTADOS E DISCUSSÃO}

Na Tabela 1 são apresentados os valores dos parâmetros de ajuste aos modelos cinéticos de pseudo $1^{\mathrm{a}}$ ordem, pseudo $2^{\mathrm{a}}$ ordem e difusão intrapartícula.

Tabela 1 - Parâmetros dos ajustes aos modelos cinéticos

\begin{tabular}{|c|c|c|}
\hline Modelo & Parâmetros & $\boldsymbol{R}^{2}$ \\
\hline Pseudo $1^{\mathrm{a}}$ ordem & $\begin{array}{c}q_{e}=14,02 \pm 0,13 \mathrm{mg} \mathrm{g}^{-1} \\
k_{l}=0,196 \pm 0,02 \mathrm{~min}^{-1}\end{array}$ & 0,948 \\
\hline Pseudo 2 $2^{\mathrm{a}}$ ordem & $\begin{array}{c}q_{e}=14,30 \pm 0,08 \mathrm{mg} \mathrm{g}^{-1} \\
k_{2}=0,036 \pm 0,003 \mathrm{~g} \mathrm{mg}^{-1} \mathrm{~min}^{-1}\end{array}$ & 0,987 \\
\hline $\begin{array}{c}C=11,66 \pm 0,33 \mathrm{mg} \mathrm{g}^{-1} \\
K_{d i f}=0,214 \pm 0,03 \mathrm{~g} \mathrm{mg} \mathrm{min}^{-0,5}\end{array}$ & 0,954 \\
\hline
\end{tabular}


Através dos dados da Tabela 1, nota-se que o modelo de pseudo $2^{\mathrm{a}}$ ordem apesentou um melhor ajuste aos dados experimentais, com um valor de $R^{2}$ de 0,98 e um valor de $q_{e}$ de 14,3 $\mathrm{mg} \mathrm{g}^{-1}$, sendo este próximo ao valor calculado de 13, $6 \mathrm{mg} \mathrm{g}^{-1}$. Segundo Ho e McKay (1999), este modelo se aplica a processos em que ocorre adsorção química.

Na Figura 2 são apresentados os ajustes cinéticos aos modelos de pseudo $1^{\mathrm{a}}$ ordem, pseudo $2^{\mathrm{a}}$ ordem e difusão intrapartícula, enquanto na Figura 3 é apresentada a frequência de distribuição dos resíduos. Tais figuras corroboram com o que foi apesentado na Tabela 1, demonstrando que a cinética de adsorção do corante azul de metileno pela cinza leve segue o modelo de pseudo $2^{\mathrm{a}}$ ordem. Ainda, pode-se observar que o modelo de difusão intrapartícula, apesar de apresentar um $R^{2}$ de 0,95 não ajustou-se satisfatoriamente. Isto pode ser explicado pelo fato de que a difusão é um processo que normalmente ocorre de forma mais lenta.

Figura 2 - qt em função do tempo com os ajustes aos modelos cinéticos.

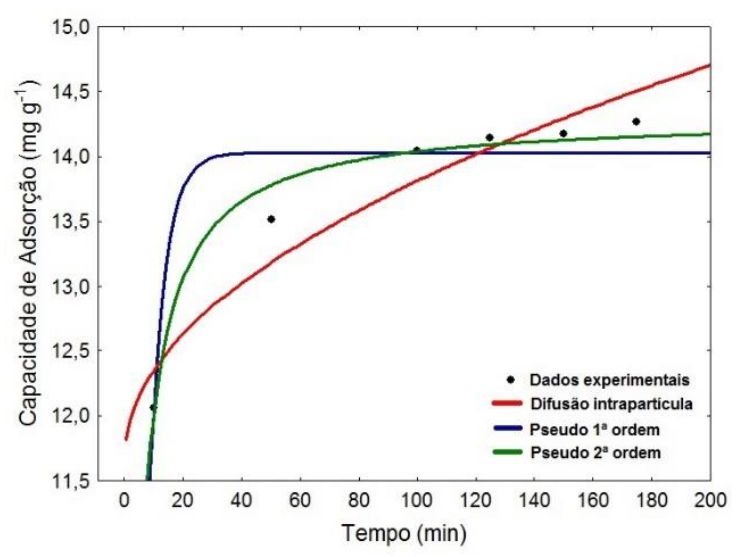

Figura 3 - Frequência de distribuição dos resíduos dos ajustes cinéticos, onde: A) pseudo $1^{\mathrm{a}}$ ordem; B) pseudo $2^{\mathrm{a}}$ ordem e C) difusão intrapartícula.

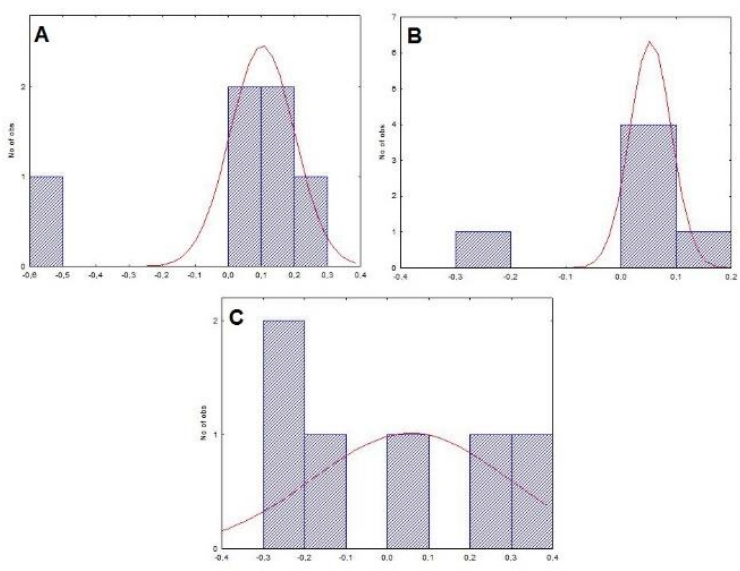

Na Tabela 2 são apresentados os parâmetros dos ajustes aos modelos de isotermas de Langmuir, Freundlich, Temkin e Sips.

Tabela 2 - Parâmetros das isotermas de adsorção

\begin{tabular}{|c|c|c|}
\hline Modelo & Parâmetros & $\boldsymbol{R}^{\mathbf{2}}$ \\
\hline Langmuir & $\begin{array}{c}q_{\text {max }}=15,06 \pm 0,63 \mathrm{mg} \mathrm{g}^{-1} \\
K_{L}=6,045 \pm 1,2 \mathrm{~L} \mathrm{mg}^{-1} \\
R_{L}=5,5 \mathrm{E}-4\end{array}$ & 0,954 \\
\hline Freundlich & $\begin{array}{c}n=9,456 \pm 1,08 \\
K_{F}=10,513 \pm 0,39 \mathrm{mg} \mathrm{g}^{-1}(\mathrm{mg} \mathrm{L}- \\
1)^{-1 / n}\end{array}$ & 0,964 \\
\hline Temkin & $\begin{array}{c}B_{T}=-2,226 \\
K_{T}=0,007 \mathrm{~L} \mathrm{~g}^{-1}\end{array}$ & 0,947 \\
\hline Sips & $\begin{array}{c}q_{\operatorname{máx}}=17,19 \mathrm{mg} \mathrm{g}^{-1} \\
K_{S}=1,962\left(\mathrm{mg} \mathrm{L}^{-1}\right)^{-1 / \mathrm{n}} \\
n_{s}=2,295\end{array}$ & 0,982 \\
\hline
\end{tabular}

Em relação ao modelo de Langmuir, um indicativo muito importante é o fator de separação $R_{L}$. Este fator indica que o processo é favorável, visto que está entre 0 e 1 (Mezzari, 2002). O valor de $n$ da isoterma de Freundlich também indica que o processo é favorável, pois 
está entre 1 e 10 (Satyanarayana et al., 1999). Já o valor de $K_{T}$ da isoterma de Temkin representa a energia máxima de ligação (Mane et al., 2007).

$\mathrm{Na}$ Figura 4, verifica-se uma grande afinidade entre a cinza e o corante, visto que inicialmente há um aumento na capacidade de adsorção, seguida de uma etapa com menor variação, indicando a proximidade da máxima capacidade de adsorção. A isoterma obtida apresenta perfil similar à do tipo I da classificação de Brunauer.

Por meio da Tabela 2, observa-se que o modelo de Sips apresentou o maior valor de correlação $\left(R^{2}=0,98\right)$ e um valor de $q_{\text {máx }}$ muito próximo ao valor calculado que foi de 16,69 $\mathrm{mg} \mathrm{g}^{-1}$. Porém, como somente a análise deste parâmetro não é suficiente para avaliar o melhor modelo, na Figura 5 são apresentadas as frequências de distribuição de resíduos para os ajustes das isotermas. Através da análise desta figura pode-se observar que as isotermas de Langmuir e Freundlich apresentaram melhores ajustes.

Figura $4-q_{t}$ em função da concentração com ajustes aos modelos de isotermas.

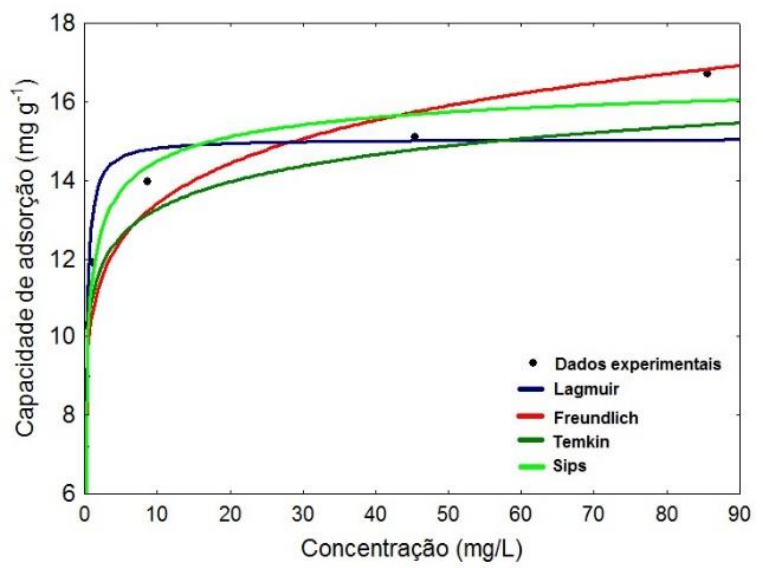

Figura 5 - Frequência de distribuição dos resíduos dos ajustes das isotermas. A) Langmuir; B) Freundlich; C) Temkin e D) Sips.
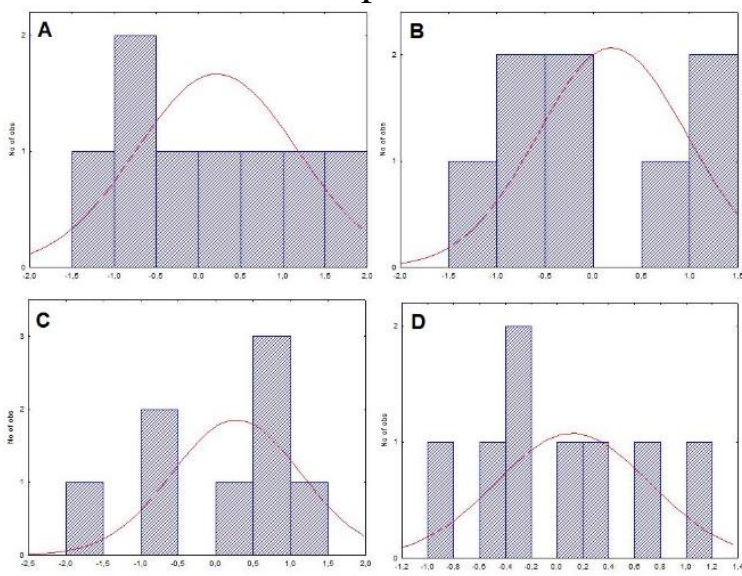

Na Tabela 3 são apresentados os valores da eficiência de adsorção do corante azul de metileno pela cinza leve, de acordo com a variação da massa utilizada. Estes valores podem ser confirmados pela Figura 6.

Tabela 3 - Eficiência de adsorção

\begin{tabular}{|c|c|c|c|}
\hline Massa (g) & Eficiência (\%) & Massa (g) & Eficiência (\%) \\
\hline 0,2 & $18,64 \pm 0,48$ & 1,2 & $99,59 \pm 0,03$ \\
\hline 0,4 & $43,43 \pm 3,10$ & 1,4 & $99,90 \pm 0,003$ \\
\hline 0,6 & $70,35 \pm 0,40$ & 1,6 & $99,89 \pm 0,05$ \\
\hline 0,8 & $84,22 \pm 0,37$ & 1,8 & $99,95 \pm 0,03$ \\
\hline 1,0 & $96,98 \pm 0,11$ & 2 & $99,94 \pm 0,007$ \\
\hline
\end{tabular}

Figura 6 - Soluções de azul de metileno após o processo de adsorção.

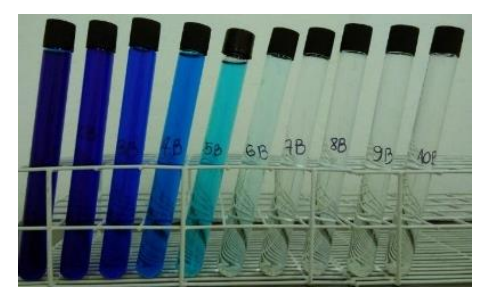

De acordo com a Tabela 3 e a Figura 6, nota-se que a cinza leve mostrou-se muito eficiente na remoção do corante azul de metileno. Os maiores valores de eficiência foram 
obtidos com as maiores massas utilizadas, sendo o maior valor de 99,95\%, com uma massa de $1,8 \mathrm{~g}$ de cinza. A Figura 6 comprova esta eficiêcia, visto que as soluções remanescentes diminuem sua coloração com o aumento da massa de cinza.

\section{CONCLUSÃO}

Os ensaios de adsorção do corante azul de metileno pela cinza leve apresentaram um melhor ajuste cinético ao modelo de pseudo $2^{\mathrm{a}}$ ordem, com um valor de $R^{2}$ de 0,98 e um valor de $q_{e}$ de $14,3 \mathrm{mg} \mathrm{g}^{-1}$, sendo este próximo ao valor calculado de $13,6 \mathrm{mg} \mathrm{g}^{-1}$, que é consideravelmente menor que o valor máximo para o carvão ativado comercial, que é de 232,6 $\mathrm{g} \mathrm{mg}^{-1}$, conforme Brum et al. (2008). Mesmo com uma capacidade de adsorção menor que a do carvão ativado comercial, a cinza leve ainda demonstra um potencial de aplicação factível devido ao seu alto volume de produção com a gaseificação do carvão e por não necessitar de processos de ativação. Para as isotermas, os modelos de Langmuir e Freundlich ajustaram-se satisfatoriamente, e ambos indicam que a adsorção é um processo favorável.

Em relação à eficiência de adsorção, constatou-se que a cinza leve é um bom adsorvente, obtendo-se uma eficiência máxima de remoção do corante de 99,95\%. Esta avaliação do potencial de adsorção é de grande importância por apresentar um possível uso deste resíduo e agregar valor ao mesmo, que é produzido em grandes volumes.

\section{NOMENCLATURA}

$B_{T} \quad$ Constante adimensional de Temkin

$C$ Constante relacionada a resistência à difusão

$k_{1} \quad$ Constante de velocidade de adsorção de $1^{\mathrm{a}}$ ordem

$\left[\mathrm{M} \mathrm{M}^{-1}\right]$

$k_{2} \quad$ Constante de velocidade de adsorção de $2^{\mathrm{a}}$ ordem

$k_{\text {dif }} \quad$ Constante de velocidade de difusão intrapartícula

$K_{L} \quad$ Constante de Langmuir

$K_{F} \quad$ Constante de Freundlich

KS Constante de Sips

$K_{T} \quad$ Constante de Temkin

$\left[\mathrm{M} \mathrm{M}^{-1} \mathrm{~T}^{-1 / 2}\right]$

$\left[\mathrm{L}^{3} \mathrm{M}^{-1}\right]$

$\left[\mathrm{M} \mathrm{M}^{-1}\right]\left[\mathrm{L}^{3} \mathrm{M}^{-1}\right]^{1 / n}$ $\left[\mathrm{L}^{3} \mathrm{M}^{-1}\right]^{1 / \mathrm{ns}}$

$n \quad$ Fator de heterogeneidade de Freundlich

$\left[\mathrm{L}^{3} \mathrm{M}^{-1}\right]$

ns Coeficiente de heterogeneidade de Sips

$q_{e} \quad$ Capacidade de adsorção

qmáx Capacidade máxima de adsorção do material

$q_{t} \quad$ Quantidade de corante adsorvido em certo instante

$R_{L} \quad$ Fator de separação de Langmuir

\section{REFERÊNCIAS}

ANEEL. Atlas da Energia Elétrica no Brasil. Brasília: Agência Nacional de Energia Elétrica, $3^{\mathrm{a}} \mathrm{ed}, 2008$.

BASU, P. Combustion and Gasification in Fluidized Beds. Cambridge: CRC Press, 2006.

BRUM, S. S.; BIANCHI, M. L.; SILVA, V. L.; GONÇALVES, M.; GUERREIRO, M. C.; OLIVEIRA, L. C. A. Preparação e caracterização de carvão ativado produzido a partir de 
resíduos do beneficiamento do café. Quím. Nova, São Paulo, v. 31, n. 5, p. 1048-1052, 2008.

CARDOSO, N. F. Remoção do corante azul de metileno de efluentes aquosos utilizando casca de pinhão in natura e carbonizada como adsorvente. 2010. Dissertação (Mestrado em química) - Universidade Federal do Rio Grande do Sul, RS. 2010.

DIEHL, R. O., FLORES, F. Q., SANTOS, F. A., CANTELLI, M. Uso de cascas para a remoção do corante azul de metileno em soluções aquosas. In: XII SALÃO DE INICIAÇÃ̃O CIENTÍFICA - PUCRS, 2011, Porto Alegre. Anais... 2011.

FUNGARO, D. A.; BRUNO, M. Utilização de zeólitas sintetizadas a partir de cinza de carvão para remoção de azul de metileno em água. Orbital the Eletronic Journal of Chemistry, v. 1, n. 1, p. 49-63, 2009.

GARCIA, Roberto. Combustíveis e combustão industrial. Interciência, 2002.

HO, Y. ; MCKAY, G. Pseudo second order model for sorption processes. Process Biochemistry, v. 34, p. 451-465, 1999.

KUNZ, A.; PERALTA-ZAMORA, P. Novas Tendências no Tratamento de Efluentes Têxteis. Química Nova, v. 25, n. 1, p. 78-82, 2002.

MANE, V. S.; DEO MALL, I.; CHANDRA SRIVASTAVA, V. Kinetic and equilibrium isotherm studies for the adsorptive removal of Brilliant Green dye from aqueous solution by rice husk ash. Journal of Environmental Management, v. 84, n. 4, p. 390-400, 2007.

MEZZARI, I. A. Utilização de carvões adsorventes para o tratamento de efluentes contendo pesticidas. 2002. Dissertação (Mestrado em Engenharia Química) - Universidade Federal de Santa Catarina, SC. 2002.

OKA, S. Fluidized Bed Combustion. New York: Marcel Dekker, 2004.

ROHDE, G. M.; MACHADO, C. S. Quantificação das cinzas de carvão fóssil produzidas no Brasil. Porto Alegre: CIENTEC, 2016.

ROYER, B. Remoção de corantes têxteis utilizando casca de semente de araucaria angustifolia como biossorvente. 2008. Dissertação (Mestrado em química) - Universidade Federal do Rio Grande do Sul, RS. 2008.

SATYANARAYANA, J.; MURTHY, G. S.; SASIDHAR, P. Adsorption studies of caesium on zirconium molybdoarsenate (ZrMAs). Waste Management, v. 19, n. 6, p. 427-432, 1999.

SMOOT, L. D.; SMITH, P. Coal Combustion and Gasification, 1985. 